\section{Three-dimensional nonlinear optical endoscopy}

\author{
Ling Fu, ${ }^{a}$ Ankur Jain, ${ }^{b}$ Charles Cranfield, ${ }^{a}$ Huikai Xie, \\ and $\operatorname{Min} \mathrm{Gu}^{\mathrm{a}, *}$ \\ ${ }^{a}$ Swinburne University of Technology, Centre for Micro- \\ Photonics, Faculty of Engineering and Industrial \\ Sciences, P. O. Box 218, Hawthorn, Victoria 3122, Australia \\ ${ }^{\mathrm{b}}$ University of Florida, Department of Electrical and \\ Computer Engineering, Gainesville, Florida 32611
}

Abstract. The development of miniaturized nonlinear optical microscopy or endoscopy is essential to complement the current imaging modalities for diagnosis and monitoring of cancers. We report on a nonlinear optical endoscope based on a double-clad photonic crystal fiber and a two-dimensional (2-D) microelectromechanical system mirror, enabling the three-dimensional (3-D) nonlinear optical imaging through in vitro gastrointestinal tract tissue and human breast cancer tissue with a penetration depth of approximately $100 \mu \mathrm{m}$ and axial resolution of $10 \mu \mathrm{m}$. The 3-D high-resolution and high-sensitive imaging ability of the nonlinear optical endoscope facilitates the visualization of 3-D morphologic and cell nuclei arrangement within tissue, and therefore will be important for histopathologic interpretation without the need of tissue excision. () 2007 Society of Photo-Optical Instrumentation Engineers. [DOI: $10.1117 / 1.2756102]$

Keywords: nonlinear optical microscopy; endoscopy; photonic crystal fiber; microelectromechanical system (MEMS) mirror; threedimensional (3-D) tissue imaging.

Paper 07021LRR received Jan. 17, 2007; revised manuscript received May 12, 2007; accepted for publication May 15, 2007; published online Jul. 16, 2007.

Disease diagnosis at an early stage requires high-resolution imaging that can visualize physiological and morphological changes at a cellular level. ${ }^{1,2}$ In particular, nonlinear optical microscopy has provided spectacular sights into visualization of cellular events within live tissues. ${ }^{3-6}$ To further extend nonlinear optical imaging for in vivo applications, miniaturized nonlinear optical microscopy and endoscopy have been exploited recently for intact organ access without tissue biopsy on a benchtop. ${ }^{2,7-18}$ Key challenges of developing a nonlinear optical endoscope are the efficient delivery of both the excitation beam and the nonlinear optical signals, the miniaturization of laser-scanning mechanisms, and the flexibility and compactness of the probe.

Since the first demonstration of miniaturized two-photon excited fluorescence (TPEF) microscopy five years ago, novel fiber-optic devices such as hollow-core photonic crystal fibers (PCFs) ${ }^{10}$ and miniaturized scanning mechanisms such as piezoelectric actuators ${ }^{7,17}$ have been adopted to overcome these technical hurdles. However, the current nonlinear optical endoscopes are not suitable for minimally invasive insertion into internal organs due to the nonflexible system

*Tel: 61-3-92148776; E-mail: mgu@swin.edu.au arrangement ${ }^{8-10}$ or the lack of sufficient sensitivity resulting from the single-mode fiber-optic devices ${ }^{13,14}$ or from a lowreflectivity microelectromechanical system (MEMS) mirror. ${ }^{11}$ Thus, the MEMS-based system ${ }^{11}$ has not been used to acquire three-dimensional (3-D) tissue imaging. In this letter, we first introduce a high-reflectivity two-dimensional (2-D) MEMS mirror to a nonlinear optical endoscope system and then report on 3-D high-resolution imaging from in vitro internal organ and cancer tissues, demonstrating the feasibility of nonlinear optical endoscopy that aims at in vivo 3-D imaging in deep tissue.

A layout of the nonlinear optical endoscope system is shown in Fig. 1(a). A double-clad PCF [Fig. 1(b)] is used to enhance the sensitivity of the endoscope system due to its large core to alleviate nonlinear optical effects for pulse delivery in the near-infrared wavelength range and high numerical aperture (NA) for signal collection in the visible wavelength range. ${ }^{15} \mathrm{~A}$ two-axis scanning mirror based on MEMS technology [Fig. 1(c)] can two-dimensionally scan the laser beam from the fiber for 2-D image formation. MEMS mirrors can facilitate endoscopic beam scanning because of their small size, high speed, low driving voltage, and excellent optical beam manipulation capability. ${ }^{11,16,19,20}$ Although a onedimensional (1-D) MEMS mirror for nonlinear optical endoscopy has been demonstrated in the prior study, ${ }^{16}$ a $2-\mathrm{D}$ MEMS mirror is required to achieve 3-D endoscopic nonlinear optical imaging. The focal plane inside tissue is altered by an external 1-D translation stage. To acquire in vivo 3-D imaging, it is necessary to integrate a 1-D MEMS actuator in the future design for axial scanning by changing the distance between the fiber and the gradient-index (GRIN) lens. ${ }^{12}$

As shown in Fig. 1(a), a pulsed laser beam at a wavelength of $800 \mathrm{~nm}$ generated from a turnkey Ti:Sapphire (Spectra Physics, MaiTai) with an $80-\mathrm{MHz}$ repetition rate and an approximately 80 -fs pulse width is launched into the doubleclad PCF through a grating pair (Newport, 1200 grooves $/ \mathrm{mm}$ ), a coupling objective, and a dichroic mirror $(\mathrm{DCM})$. The coupling objective (CO, Melles Griot, $4 \times / 0.12 \mathrm{NA})$ is chosen to maximize the excitation power delivered by the fiber core. The endoscope probe consists of a port of the double-clad PCF, a 2-D MEMS mirror, and a GRIN lens (Melles Griot, $1.8 \mathrm{~mm}$ diam, 0.6 NA, 0.23 pitch), allowing an outer diameter of $5 \mathrm{~mm}$ and a working distance of approximately $200 \mu \mathrm{m}$.

The 2-D MEMS mirror we used is based on electrothermal actuation and can perform large bidirectional 2-D optical scans over $\pm 30 \mathrm{deg}$ at less than $12 \mathrm{Vdc}^{21}$ The aluminumcoated mirror plate is $0.5 \mathrm{~mm}$ by $0.5 \mathrm{~mm}$ in size and has a reflection efficiency of approximately $80 \%$ at wavelength $800 \mathrm{~nm}$. Furthermore, the small initial tilt angle of the mirror simplifies the endoscope design and packaging. Two actuators of the mirror are synchronized to create a raster scanning pattern by applying 2.5 to $7.5 \mathrm{Vdc}$ voltages. The scanning rate of the mirror is 7 lines/s for our experiments, although its resonance frequency is approximately $480 \mathrm{~Hz}$, which could be used to increase the speed for imaging acquisition. It should be pointed out that the low driving voltage and the ruggedness of operation make this 2-D MEMS mirror suitable and safe for clinical applications.

1083-3668/2007/12(4)/040501/3/\$25.00 @ 2007 SPIE 


\section{JBO LETTERS}
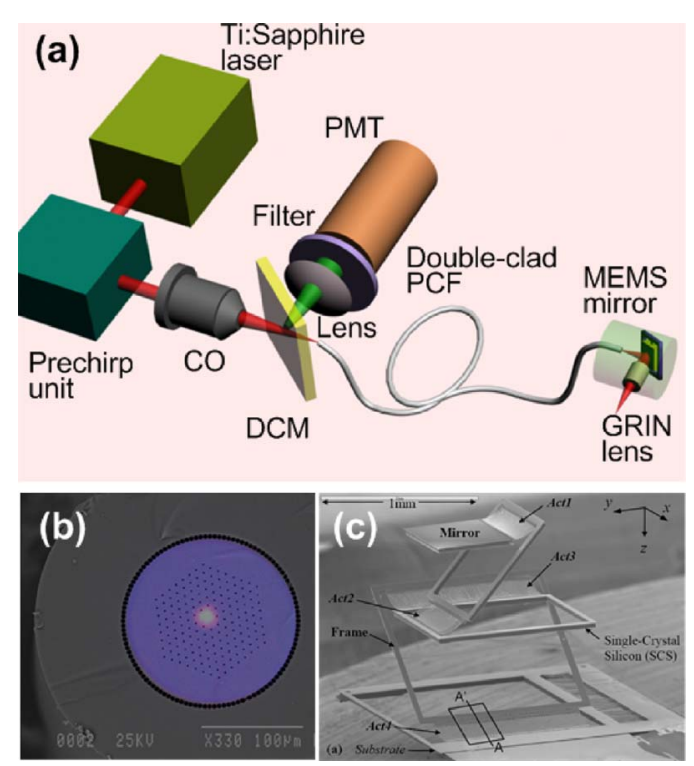

Fig. 1 (a) The nonlinear optical endoscope system using a doubleclad PCF, a 2-D MEMS mirror, and a GRIN lens. (b) Overlay of scanning electron microscopy (SEM) image of a double-clad PCF and the output patterns at wavelengths $410 \mathrm{~nm}$ and $800 \mathrm{~nm}$ shows the singlemode delivery in the fiber core at a near-infrared wavelength and the multimode propagation of visible light through the inner cladding. (c) SEM image of the MEMS mirror. The entire size is $2.7 \mathrm{~mm} \times 1.9 \mathrm{~mm}$ $\times 1.2 \mathrm{~mm}$.

The imaging capability of the nonlinear optical endoscope is demonstrated by the TPEF imaging with $10-\mu \mathrm{m}$-diam fluorescent microspheres [Fig. 2(a)]. The high sensitivity of the system is also confirmed by the comparison between this image and the TPEF image [Fig. 2(b)] obtained from a system based on a single-mode fiber coupler, a GRIN lens, and a bulk scanning stage. ${ }^{22}$ It is shown that the signal level in the former
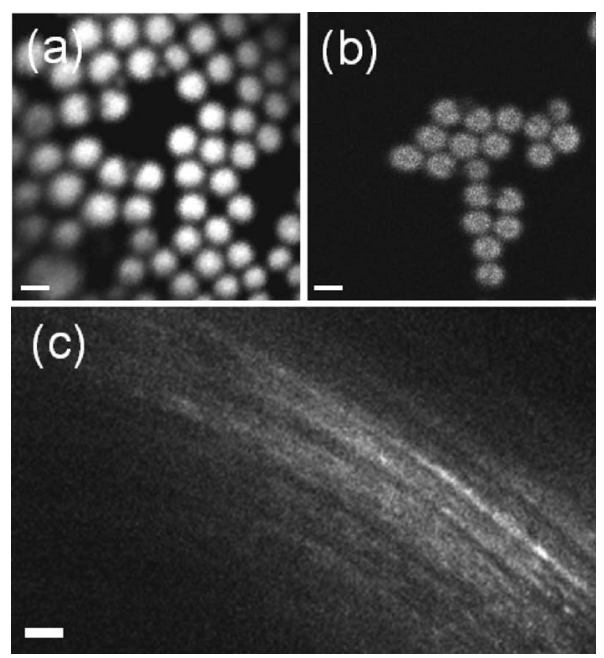

Fig. 2 TPEF images obtained (a) through a double-clad PCF, a 2-D MEMS mirror, and a GRIN lens and (b) through a single-mode fiber coupler, a GRIN lens, and a bulk scanning stage. The sample is fluorescent microspheres with a diameter of $10 \mu \mathrm{m}$. The power in (a) for TPEF is approximately $1.8 \mathrm{~mW}$. (c) $Z$ projection of $11 \mathrm{SHG}$ sections obtained from the rat tail tendon with an imaging spacing of $10 \mu \mathrm{m}$. Scale bars are $10 \mu \mathrm{m}$.

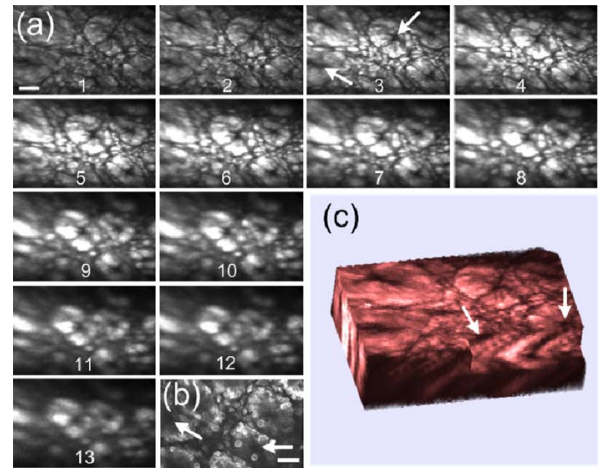

Fig. 3 (a) A series of in vitro images of rat large intestine tissue. Each section is recorded at an axial step of $7.5 \mu \mathrm{m}$ into the sample. The excitation power is approximately $40 \mathrm{~mW}$ at wavelength $800 \mathrm{~nm}$. (b) A TPEF image of the rat large intestine tissue taken from a standard laser scanning microscope (Olympus, Fluoview) with an objective (Olympus, UplanApo, $40 \times / 0.85$ NA). (c) 3-D visualization of the rat large intestine tissue based on the image stack in (a). Image reconstruction is performed using AMIRA (Mercury Computer Systems). Arrows point to the intestinal crypts. Scale bars are $20 \mu \mathrm{m}$.

case is approximately 120 times higher than that in the latter due to the large collection area and the high NA of the inner cladding of the PCF. Furthermore, combined with the previous characterization experiment in which case a MEMS mirror is not used and the focal spot is the same, ${ }^{16}$ the result in Fig. 2(a) implies that 80 -fs pulses are broadened by approximately $25 \%$ after the MEMS mirror, as the TPEF efficiency is inversely proportional to the pulse width. ${ }^{23}$ The pulse broadening might be due to the group-velocity dispersion caused by the aluminum coating of the mirror plate. The ability of second harmonic generation (SHG) imaging through the endoscope system is shown by SHG optical sections from a rat tail tendon [Fig. 2(c)], which is dissected from a Sprague-Dawley rat and imaged directly.

Since the majority of cancers are epithelial tissues in origin, we have focused our efforts on 3-D imaging of epithelial tissues. Figure 3(a) is a series of in vitro TPEF images of rat large intestine tissue. The large intestine is extracted from a Sprague-Dawley rat and stained with $1 \%$ Acridine Orange (Sigma) in Ringer's solution to enhance the image contrast. TPEF images are obtained through the thick tissue with a penetration depth of $100 \mu \mathrm{m}$. The ability of the endoscope system to discriminate the microscopic anatomy is also investigated by comparing these measurements with the TPEF image [Fig. 3(b)], which is taken from the same large intestine tissue in a standard laser-scanning TPEF microscope (Olympus, Fluoview) with a 0.85 NA objective. It is shown that the image pattern obtained from both the endoscope system and the standard microscope are similar, and surface epithelial cells surrounding intestinal crypts [see arrows in frame 3 and Fig. 3(b)] can clearly be observed in both cases. The structural details in the rat large intestine tissue can be further visualized in the 3-D reconstruction of the image stack [Fig. 3(c)], where the intestinal crypts are displayed (see arrows) by cropping the 3-D volume. In addition, the endoscope system also enables the visualization of the openings to the gastric pits of the rat stomach tissue (data not shown), demonstrating the feasibility to differentiate various tissue types and identify early mucosal lesions in the gastrointestinal tract. 


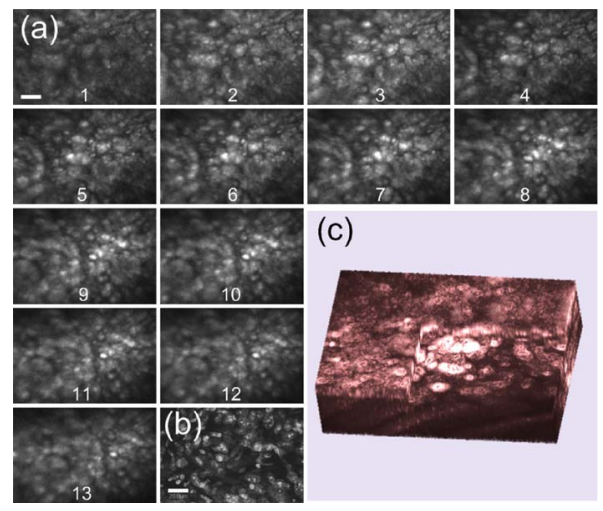

Fig. 4 (a) A series of in vitro images of the human u-87 MG glioblastoma tissue. Each section is recorded at an axial step of $5 \mu \mathrm{m}$ into the sample. The excitation power is approximately $40 \mathrm{~mW}$ at wavelength $800 \mathrm{~nm}$. (b) A TPEF image of the breast cancer tissue from the standard microscope used in Fig. 3(b). (c) 3-D visualization of the breast cancer tissue. Scale bars are $20 \mu \mathrm{m}$.

To demonstrate the imaging ability of the nonlinear optical endoscope for cancer tissue, human u-87 MG glioblastoma cells (a kind of human breast cancer cells) are xenografted into the hind leg of a nude Balb c mouse and cultured for one week before dissection from the nude mouse for imaging. The breast cancer tissue is stained with $1 \%$ Acridine Orange to visualize the cell nuclei. TPEF images of human u-87 MG glioblastoma tissue are taken from the endoscope system [Fig. 4(a)] with a penetration depth of approximately $75 \mu \mathrm{m}$ and the standard microscope [Fig. 4(b)], respectively. Figure 4(c) is the 3-D reconstruction of the TPEF images in Fig. 4(a). It is found that the endoscope system and the standard microscope produce a consistent pattern with the same sample. Furthermore, the contrast pattern of the cancer tissue is fundamentally different from that achieved in gastrointestinal tract tissue, showing the extremely dense distribution of cell nuclei in the cancer tissue.

In conclusion, a 2-D MEMS mirror has been first introduced to a nonlinear optical endoscope system that is incorporated with a double-clad PCF and a GRIN lens to demonstrate its effectiveness for flexible nonlinear optical imaging. In vitro 3-D images of internal organ tissue and cancer tissue have been achieved with a penetration depth of up to $100 \mu \mathrm{m}$ and axial resolution of approximately $10 \mu \mathrm{m}$. Epithelial cells and intestinal crypts in 3-D images from rat large intestine tissue are clearly identified. Morphological details in the breast cancer tissue are also provided by the visualization of cell nuclei. Taking advantage of compact size, high sensitivity, and high 3-D resolution, nonlinear optical endoscopy has demonstrated its potential to complement other optical imaging modalities such as optical coherent tomography and spectrally encoded endoscopy ${ }^{1}$ for in vivo imaging in biomedical applications.

\section{Acknowledgments}

This work is supported by the Australian Research Council and partially by the U.S. National Science Foundation (Grant No. 0423557). The authors acknowledge useful discussions with Sarah Russell and Carleen Cullinane at Peter MacCallum Cancer Centre.

\section{References}

1. D. Yelin, I. Rizvi, W. M. White, J. T. Motz, T. Hasan, B. E. Bouma, and G. J. Terney, "Three-dimensional miniature endoscopy," Nature (London) 443, 765 (2006).

2. B. A. Flusberg, E. D. Cocker, W. Piyawattanametha, J. C. Jung, E. L. M. Cheung, and M. J. Schnitzer, "Fiber-optic fluorescence imaging," Nat. Methods 2, 941-950 (2005).

3. F. Helmchen and W. Denk, "Deep tissue two-photon microscopy," Nat. Methods 2, 932-940 (2005).

4. P. J. Campagnola and L. M. Loew, "Second harmonic imaging microscopy for visualizing biomolecular arrays in cells, tissues and organisms," Nat. Biotechnol. 21, 1356-1360 (2003).

5. A. Zoumi, A. Yeh, and B. J. Tromberg, "Imaging cells and extracellular matrix in vivo by using second-harmonic generation and twophoton excited fluorescence," Proc. Natl. Acad. Sci. U.S.A. 99, 11014-11019 (2002).

6. W. E. Zipfel, R. M. Williams, R. Christie, A. Y. Nikitin, B. T. Hyman, and W. W. Webb, "Live tissue intrinsic emission microscopy using multiphoton-excited native fluorescence and second harmonic generation," Proc. Natl. Acad. Sci. U.S.A. 100, 7075-7080 (2003).

7. F. Helmchen, M. S. Fee, D. W. Tank, and W. Denk, "A miniature head-mounted two-photon microscope: high-resolution brain imaging in freely moving animals," Neuron 31, 903-912 (2001).

8. J. C. Jung and M. J. Schnitzer, "Multiphoton endoscopy," Opt. Lett. 28, 902-904 (2003).

9. M. J. Levene, D. A. Dombeck, K. A. Kasischke, R. P. Molloy, and W. W. Webb, "In vivo multiphoton microscopy of deep brain tissue," $J$. Neurophysiol. 91, 1908-1912 (2004).

10. B. A. Flusberg, J. C. Jung, E. D. Cocker, E. P. Anderson, and M. J. Schnitzer, "In vivo brain imaging using a portable 3.9 gram twophoton fluorescence microendoscope," Opt. Lett. 30, 2272-2274 (2005).

11. W. Piyawattanametha, R. P. J. Barretto, T. H. Ko, B. A. Flusberg, E. D. Cocker, H. Ra, D. Lee, O. Solgaard, and M. J. Schnitzer, "Fastscanning two-photon fluorescence imaging based on a microelectromechanical systems two-dimensional scanning mirror," Opt. Lett. 31, 2018-2020 (2006)

12. H. Choi, S. Chen, D. Kim, L. Munro, M. Culpepper, and P. So, "In vitro imaging of mouse colorectal tissue by nonlinear microendoscope biopsy probe," in Endoscopic Microscopy II, G. J. Tearney and T. D. Wang, Eds., Proc. SPIE 6432 (2007).

13. D. Bird and M. Gu, "Two-photon fluorescence endoscopy with a micro-optic scanning head," Opt. Lett. 28, 1552-1554 (2003).

14. W. Göbel, J. N. D. Kerr, A. Nimmerjahn, and F. Helmchen, "Miniaturized two-photon microscope based on a flexible coherent fiber bundle and a gradient-index lens objective," Opt. Lett. 29, 2521-2523 (2004).

15. L. Fu, X. Gan, and M. Gu, "Nonlinear optical microscopy based on double-clad photonic crystal fibers," Opt. Express 13, 5528-5534 (2005).

16. L. Fu, A. Jain, H. Xie, C. Cranfield, and M. Gu, "Nonlinear optical endoscopy based on a double-clad photonic crystal fiber and a MEMS mirror," Opt. Express 14, 1027-1032 (2006).

17. M. T. Myaing, D. J. MacDonald, and X. Li, "Fiber-optic scanning two-photon fluorescence endoscope," Opt. Lett. 31, 1076-1078 (2006).

18. K. König, "Clinical two-photon microendoscopy," Microsc. Res. Tech. 70, 398-402 (2007).

19. H. Xie, Y. Pan, and G. K. Fedder, "Endoscopic optical coherence tomographic imaging with a CMOS-MEMS micromirror," Sens. Actuators, A 103, 237-241 (2003).

20. K. C. Maitland, H. J. Shin, H. Ra, D. Lee, O. Solgaard, and R. Richards-Kortum, "Single fiber confocal microscope with a two-axis gimbaled MEMS scanner for cellular imaging," Opt. Express 14, 8604-8612 (2006).

21. A. Jain and H. Xie, "An electrothermal SCS micromirror for large bi-directional 2D scanning," in Proc. 13th International Conference on Solid-state Sensor, Actuators, and Microsystems, 988-991 (2005).

22. L. Fu, X. Gan, and M. Gu, "Characterization of the GRIN lens-fiber spacing toward applications in two-photon fluorescence endoscopy," Appl. Opt. 44, 7270-7274 (2005).

23. W. Denk, J. H. Strickler, and W. W. Webb, "Two-photon laser scanning fluorescence microscopy," Science 248, 73-75 (1990). 